\title{
CONHECIMENTO CLÍNICO DO ENFERMEIRO NA ATENÇÃO PRIMÁRIA À SAÚDE: APLICAÇÃO DE UMA MATRIZ FILOSÓFICA DE ANÁLISE ${ }^{1}$
}

\author{
Marta Regina Cezar-Vaz², Letícia Silveira Cardoso33, Clarice Alves Bonow ${ }^{4}$, Cynthia Fontella Sant'Anna \\ Janaina Sena ${ }^{6}$
}

\begin{abstract}
${ }^{1}$ Parte do relatório de pesquisa - Trabalho em Saúde e o Contexto Tecnológico da Política de Atenção à Saúde da Família, desenvolvida com financiamento do MS/CNPq/FAPERGS No 008/2004/2007- Programa Pesquisa para o SUS - sob o Processo $\mathrm{N}^{\circ} 0415374$.

${ }^{2}$ Doutora em Filosofia da Enfermagem. Professor Associado da Escola de Enfermagem da Universidade Federal do Rio Grande (FURG). Rio Grande do Sul, Brasil. E-mail: cezarvaz@vetorial.net

${ }^{3}$ Mestranda em Ciências da Saúde da FURG. Bolsista CAPES. Rio Grande do Sul, Brasil. E-mail: lsc_enf@yahoo.com.br

${ }^{4}$ Mestranda em Ciências da Saúde da FURG. Professor Temporário da Escola de Enfermagem da FURG. Rio Grande do Sul, Brasil. E-mail: enfcla@yahoo.com.br

${ }^{5}$ Mestranda em Enfermagem da FURG. Bolsista CAPES. Rio Grande do Sul, Brasil. E-mail: cynthiafs_enf@yahoo.com.br

${ }^{6}$ Doutoranda em Educação Ambiental da FURG. Professor Assistente da Escola de Enfermagem da FURG. Rio Grande do Sul, Brasil. E-mail: janaenf@hotmail.com
\end{abstract}

\begin{abstract}
RESUMO: Neste estudo analisa-se a constituição do conhecimento clínico que orienta a prática do enfermeiro, na Atenção Primária à Saúde, para operar no objeto/sujeito da ação. Estudo exploratório com abordagem qualitativa e análise temática dos dados. Foram realizadas entrevistas semi-estruturadas com 65 enfermeiros da rede pública de saúde da família, no extremo sul do Rio Grande do Sul, Brasil. Os dados foram analisados a partir de uma matriz que sustenta a ciência clínica da enfermagem em uma base populacional, constituída por três núcleos - o indivíduo e a família, a comunidade e o sistema de saúde. Evidenciou-se que o conhecimento clínico organiza a prática do enfermeiro com o foco predominante no indivíduo e na família. Sem, contudo, subsumir à sua raiz de origem a base populacional, por meio da forma como o conhecimento clínico faz apreender/conhecer o objeto da ação. Acredita-se que tais achados possam subsidiar uma releitura do potencial organizativo do conhecimento clínico da enfermagem.
\end{abstract}

DESCRITORES: Atenção primária à saúde. Serviços de saúde. Enfermagem em saúde pública.

\section{NURSE CLINICAL KNOWLEDGE IN THE HEALTH PRIMARY ATTENTION: APPLICATION OF AN ANALYSIS PHILOSOPHICAL MATRIX}

\begin{abstract}
In the present study, the constitution of the clinical knowledge which guides the nurse practice, in the health primary attention, is analyzed, in order to use it on the object / subject of the action. Exploratory study with qualitative approach and thematic analysi of data. Semi-structured interviews were made with 65 nurses from the family health public services, in te southern part of Rio Grande do Sul state, Brazil. The data was analyzed based on a matrix which sustains the nursing clinical science in a populational basis, constituted by three topics - the individual and the family, the community and the health system. It was made clear that the clinical knowledge organizes the nurse practice in the predominant focus of the individual and the family. Without, however, connecting to the origin - the populational base, through the way the clinical knowledge enables learning/knowing the object of the action. It is believed that these findings may subsidize a new reading of the organizational potential of the nursing clinical knowledge.
\end{abstract}

DESCRIPTORS: Primary health care. Health services. Public health nursing.

\section{EL CONOCIMIENTO CLÍNICO DEL ENFERMERO EN LA ATENCIÓN PRIMARIA DE LA SALUD: APLICACIÓN DE UNA MATRIZ DE ANÁLISIS FILOSÓFICO}

\begin{abstract}
RESUMEN: En este estudio se analiza la constitución del conocimiento clínico que guía la práctica del enfermero en la Atención Primaria de la Salud, para el funcionamiento del objeto/sujeto de la acción. Es un estudio exploratorio con enfoque cualitativo y análisis temático de los datos. Se realizaron entrevistas semiestructuradas con 65 enfermeros del área de salud pública familiar, en el sur de Rio Grande do Sul, Brasil. Los datos fueron analizados utilizando una matriz que se apoya en la ciencia clínica de la enfermería con base en la población, constituída por tres núcleos: el individuo y la familia, la comunidad, y el sistema de salud. Se ha mostrado que el conocimiento clínico organiza la práctica del enfermero con enfoque predominante en el individuo y la familia. Sin subsumir la raíz de su origen, la población, por medio del conocimiento clínico se hace aprender/conocer el objeto de la acción. Se cree que estos descubrimientos pueden apoyar una reinterpretación de las posibilidades de organización de los conocimientos clínicos de enfermería.
\end{abstract}

DESCRIPTORES: Atención primaria de salud. Servicios de salud. Enfermería en salud pública. 


\section{INTRODUÇÃO}

O presente estudo tem como pressuposto o fato de o conhecimento clínico utilizado pelo enfermeiro no trabalho na Atenção Primária à Saúde, ${ }^{1}$ na particularidade Saúde da Família (SF), estar centrado no objeto de sua ação. Esse conhecimento é próprio do núcleo da enfermagem. O núcleo do conhecimento clínico é o indivíduo, tanto para os enfermeiros atuantes na rede de atenção hospitalar quanto para aqueles da rede de atenção primária à saúde. $\mathrm{O}$ que difere no referido núcleo, então? O diferente é o próprio objeto/sujeito a que se quer conhecer/apreender, por meio da organização do conhecimento, a qual será condicionada por ele. E o trabalhador organiza a ação, por meio do conhecimento clínico condicionado por àquele objeto/sujeito.

O objeto da ação do enfermeiro é construído por meio do conhecimento da enfermagem e esse se configura de maneira diferente já na própria organização do conhecimento, no qual não é suficiente a organização em termos de um conhecimento bio-orgânico para transformar àquele objeto da ação em produto da sua própria satisfação e, ao mesmo tempo, produto da satisfação da prática da enfermagem.

Se a organização do conhecimento posto é a clínica e sua prática, ou mesmo, a prática clínica, ${ }^{2-3}$ significa dizer que, ao lhe ser atribuído uma objetividade limite, pode-se descobrir outra ordem que não se inclui nela. O fato está na convicção de que a clínica do corpo não é a mesma para o enfermeiro da atenção primária à saúde e do enfermeiro de outro trabalho. Sabe-se que a prática clínica geral apreende o seu objeto tangenciando o conhecimento do corpo humano. A prática (ou práticas) em questão reconhece/atua a partir da organização de seu conhecer sobre o limite do corpo orgânico, em outras palavras, no limite da organização do conhecimento possível de sua clínica, anunciado pela descoberta do agravo/alteração e da possível doença orgânica (mental ou física) dentro de matrizes organizacionais de conhecimento.

O aparente acréscimo é provocado pela necessidade de apreensão e transformação do objeto, que é o sujeito da ação prática. Aparente acréscimo ou somatório porque tais componentes estão na raiz da construção do conhecimento clínico do enfermeiro da rede de Atenção Primária à Saúde. E, partindo de tal pressuposto reitera-se que esses componentes encontram-se comprimidos na própria raiz do conhecimento e confirma-se que é o objeto a conhecer sob deter- minada organização do conhecimento e nenhum outro que diferencia a aplicação do conhecimento clínico de um ou de outro profissional, no caso presente, o foco do enfermeiro.

Assumir a mencionada perspectiva é fortalecer o conhecimento clínico, ${ }^{1,4}$ na perspectiva teórica e filosófica de que a ciência da enfermagem é orientadora da prática e mesma prática aciona a produção de seu conhecimento. Eis o contexto teórico, filosófico e empírico motivador da presente investigação, a qual tem como objetivo analisar a constituição do conhecimento clínico que orienta a prática do enfermeiro na atenção primária à saúde, na particularidade dos enfermeiros atuantes na rede pública de SF. E, também compreender a partir da análise proposta as formas como o conteúdo da clínica se desenvolve na ação sobre o objeto/sujeito da ação. Diante do que foi exposto, acredita-se que a presente pesquisa possa ser um instrumento contributivo para a ciência da enfermagem.

\section{METODOLOGIA}

A pesquisa se caracteriza por ser um estudo transversal com abordagem dialética ${ }^{5}$ a qual congrega a análise qualitativa temática ${ }^{6}$ da constituição do conhecimento clínico à constituição que orienta a prática do enfermeiro na Atenção Primária à Saúde, na particularidade dos enfermeiros atuantes na rede pública de SF. E, também compreender, a partir da análise procedida, as formas como o conteúdo da clínica (a prática clínica) se desenvolve na ação sobre o objeto/sujeito da ação.

O cenário do estudo compreendeu a rede pública de atenção à SF da Terceira Coordenadoria Regional de Saúde, no extremo sul do Rio Grande do Sul, Brasil. A população da pesquisa constituise de 65 enfermeiros que compõem a totalidade de profissionais da categoria atuantes nas 65 equipes da SF existentes no semestre que antecedeu a coleta dos dados, realizada entre os meses de janeiro a julho de 2006, através de entrevistas semiestruturadas gravadas. Destaca-se que foi utilizado como critério de seleção das equipes a constituição das mesmas até o período de 2004, ano em que foi aprovado o projeto de pesquisa.

O roteiro da entrevista foi testado por meio de estudo piloto junto a uma equipe de saúde da família não pertencente ao grupo selecionado para a amostra, e estruturado em duas partes: a primeira, um questionário para caracterização dos sujeitos (sexo, idade, formação); e a segunda, 
o roteiro propriamente dito das entrevistas que, para a presente apresentação, orientou-se através das seguintes questões norteadoras: Como o conhecimento clínico é constituído na prática do enfermeiro na Atenção Primária à Saúde, na particularidade da SF? Como a prática clínica do enfermeiro se desenvolve na ação sobre o objeto/ sujeito da ação?

As entrevistas foram digitalizadas e analisadas no programa NVivo 7.0. Com o auxílio dessa ferramenta construiu-se uma malha (árvore) de análise qualitativa do tema. ${ }^{6}$ Para tanto, utilizou-se as categorias definidas a priori, do quadro teórico e filosófico ${ }^{7}$ do conhecimento da enfermagem, particularmente, no caso do estudo proposto, o conhecimento clínico e sua organização prática (a prática clínica), na Atenção Primária à Saúde; do pressuposto filosófico de que a prática do enfermeiro é orientada pelo conhecimento gerado no núcleo da enfermagem, que organiza o conhecimento para conhecer e apreender seu objeto da ação prática. E, na especificidade da enfermagem da Atenção Primária à Saúde, o núcleo possui em sua raiz uma base populacional, cuja constituição apreende o indivíduo e a família, a comunidade e o sistema de saúde. ${ }^{1}$ Outras categorias definidas a posteriori, a partir da própria análise temática apoiada nos dados e subsidiada na literatura, ${ }^{1,8-9}$ foram: o núcleo operatório do conhecimento clínico; a forma de organizar o conhecimento clínico para apreender o objeto/sujeito da ação (Figura 1). Salienta-se que em um mesmo depoimento foi-se buscar, em esgotamento do conteúdo, as possíveis formas de sentidos/significados do conhecimento clínico organizador da prática do enfermeiro na atenção primária à saúde. Dessa forma, são apresentadas frequências quantitativas que evidenciam a magnitude para mais ou para menos da referência ao objeto do estudo.

A proposta de pesquisa foi aprovada pelo Comitê de Ética em Pesquisa na Área da Saúde da Universidade Federal do Rio Grande, com parecer $\mathrm{N}^{\circ} 02 / 2004$. Todos os entrevistados manifestaram concordância em participar do estudo assinando em duas vias o consentimento pós-informado. Os princípios éticos foram respeitados conforme Resolução No 196/96 do Conselho Nacional de Saúde.

Tentar-se-á demonstrar por meio das argumentações-reflexivas, utilizando exemplos do cotidiano da prática, narrados pelos enfermeiros entrevistados, sem, contudo, apresentar o dado bruto. Uma tentativa não no sentido do descaso, da "pouca importância", mas pelo próprio pres- suposto que orienta a posição teórico-filosófica assumida no presente texto, que reconhece na organização do conhecimento de determinado objeto, a objetividade científica do conhecimento e, ao mesmo tempo, a subjetividade desse objeto do conhecimento, obtendo-se uma abertura de outra ordem dela, da realidade prática. Por outro lado, na organização do conhecimento em questão, existe ele próprio, o sujeito que conhece, que também assume sua objetividade e sua subjetividade como factuais.

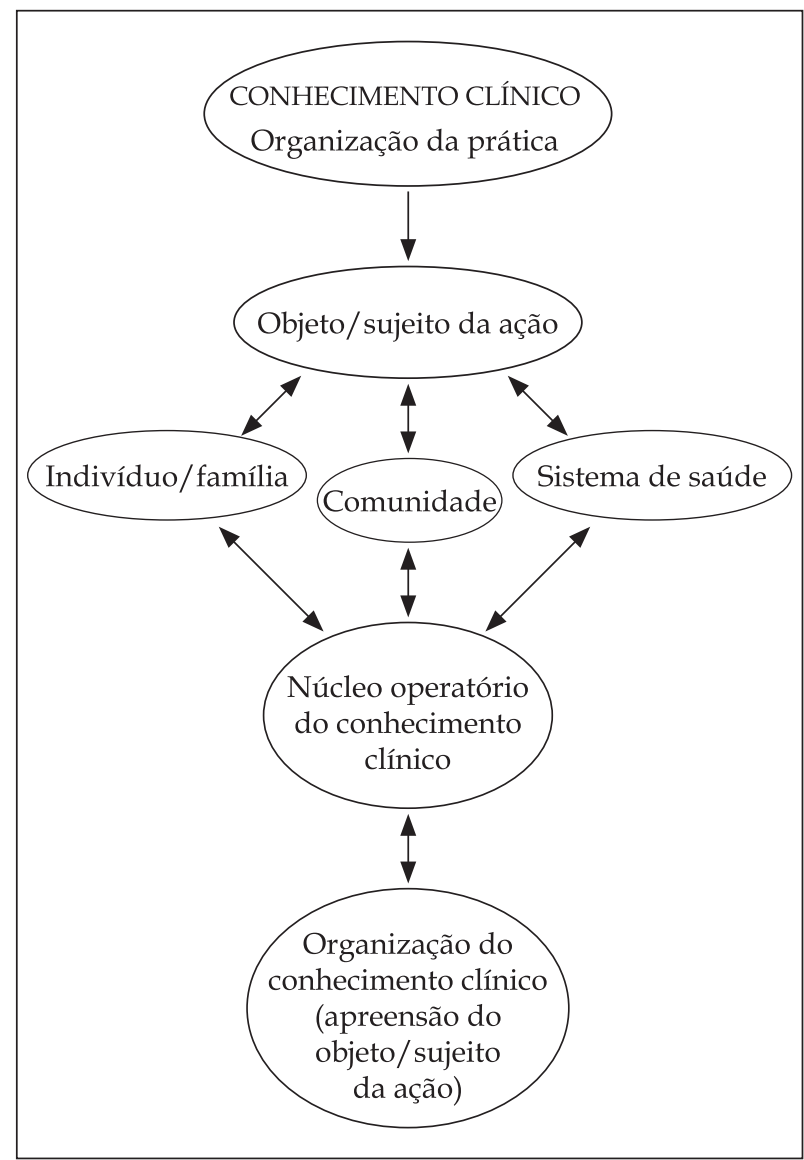

Figura 1 - Matriz da análise dos dados

\section{RESULTADOS}

A pesquisa foi composta por 65 enfermeiros, destes $59(90,77 \%)$ eram do sexo feminino e, seis $(9,23 \%)$ do sexo masculino. A média de idade foi de 37,60 \pm 7,43 (Média \pm Desvio Padrão), variando entre 24 e 55 anos de idade, sendo que, do total de trabalhadores, um ignorou a resposta. Em relação ao tempo de trabalho dos enfermeiros em Unidade Básica de Saúde da Família (UBSF) foi de 25,93 $\pm 17,22$ (Média \pm Desvio Padrão), variando entre um mês e 60 meses de atuação na UBSF; no item estudado, dois trabalhadores ignoraram a resposta. 
Do total de 65 trabalhadores, 50 (76,92\%) possuem Especialização em Saúde da Família e, 15 (23,08\%) possuem Especialização na área de Saúde Pública e Saúde Coletiva. No que se refere à formação pósgraduada stricto sensu, quatro $(6,15 \%)$ enfermeiros possuem mestrado.

Iniciando a apresentação dos resultados, na referência do conteúdo analisado das narrativas dos entrevistados, pode-se identificar uma predominância quantitativa para o indivíduo e a família, como objeto da ação de conhecer/apreender, por meio do conhecimento clínico com base populacional. No conteúdo dos 65 depoimentos, encontrou-se 164 vezes a referência dessa prática clínica pesquisada, centrada na individualidade da pessoa, seja na forma da consultação, do aconselhamento ou da pré-consulta. Nesse conjunto, as áreas temáticas de atuação encontradas no conteúdo analisado foram, em frequência decrescente, a saúde da mulher, da criança, do adulto, do idoso e do adolescente.

Ainda no foco com o qual vimos trabalhando, no que concerne à família, a frequência foi de 33 vezes, como objeto da ação apreendido por meio desse conhecimento sendo citadas as mesmas áreas de atuação, apresentadas logo acima, diferindo quanto à predominância quantitativa nos depoimentos para a saúde do adulto sem condições de deslocamento até a unidade de saúde. A família como foco do conhecimento clínico e apreensão como objeto da ação prática, aparece nos depoimentos na relação primeira com o ambiente físico familiar, a moradia. Salienta-se que o foco na família possui o sentido de auxiliar a família (companheira, companheiro, mãe, avó, por exemplo) que possua alguém situação aguda ou crônica (pacientes pós-cirúrgicos, pessoas acamadas com úlcera de decúbito, paciente ostomizado), ou com menor frequência, na área da saúde da mulher e da criança (na fase do puerpério, por exemplo).

Os achados aqui apresentados permitem evidenciar que a prática clínica com foco na família está centrada no indivíduo que requer cuidados especiais, denotando ser ele, por sua vez, o foco que provoca a apreensão da família, como objeto/sujeito da prática clínica. O que evidencia um prolongamento da prática clínica, daquela realizada na unidade, na qual a atenção é para o indivíduo, familiar da família. Tal prática clínica, estendida ao domicílio, não reduz a família como parte de um dos componentes do objeto da ação clínica do enfermeiro.

O foco no indivíduo e na família como objetos quase inseparáveis da prática clínica, segundo os achados, identificou uma predominância na forma de organizar a ação, qual seja, uma ação organizativa em torno da prevenção de uma doença ou agravo e da diminuição do sofrimento individual e familiar. Neste foco, a família é a possibilidade de ampliação da ação clínica do enfermeiro, mesmo em situação de uma doença em particular. A família estimula a ação para um coletivo que está vinculado ao sujeito/objeto individual da ação, porém se constituí em algo diferente do foco orgânico e patológico. Porquanto, no conteúdo analisado, os enfermeiros assumem o conhecimento clínico, na forma de prevenir (em relação à forma de apreensão/conhecimento do objeto da ação), como uma prática clínica valorada e diferente daquela centralizada somente na cura das doenças, ou seja, a relação, construída no dia a dia do trabalho, permitindo a permanência de um status operacional e ideal que revela a potência de poder aplicar o conhecimento clínico, no campo da saúde pública, com o sentido de diminuir possibilidades negativas de estados de adoecimento biossocial. ${ }^{8}$

No vínculo operacional formado entre a prática clínica e o foco no indivíduo e na família, o significado de atenção sobre o corpo biossocial pode salientar um marcador teórico-operacional da vigilância à saúde ${ }^{10}$ relacionado com o outro foco da raiz do conhecimento clínico, com basepopulacional, no modelo de análise aplicado - o Sistema de Saúde da Atenção Primária. O marcador a que estamos nos referindo organiza a rede de atenção, por meio de processos de identificação, priorização e intervenção nos condicionantes que podem desencadear uma enfermidade nas dimensões do indivíduo e da família e também da comunidade. Assim, a ação de conhecer/apreender o objeto da prática clínica do enfermeiro ganha abrangência coletiva.

Em síntese, mas não na redução de sentido, o conhecimento clínico que organiza a apreensão/ conhecimento do objeto da ação, a prática clínica, se mostrou com abrangência coletiva, conforme já foi referido, ou seja, os enfermeiros, ao agirem por meio do conhecimento clínico, fazem-no sobre o risco do adoecimento dentro de um espectro amplo, a abranger o campo interdisciplinar da saúde pública. Isto não significa que o enfermeiro perca de foco o indivíduo e a família como dimensão predominante na sua prática clínica.

No cenário desta pesquisa em particular, é possível defender a existência de um forte indício de que o conhecimento clínico organiza a prática 
para apreensão no risco de adoecer, no sentido de carência ou falta de condições adequadas para um bem-estar não apenas no sentido orgânico, mas social. No conteúdo das entrevistas, o risco social está relacionado aos estilos culturais de vida, os quais incluem as condições e situações de pessoas nas comunidades que convivem com o potencial do adoecimento e do sofrimento físico e emocional, predominante para ambos.

A prática clínica também aparece frequentemente, nas narrativas dos entrevistados, associada às necessidades de oferecer conhecimentos e informações para a pessoa e a família, nas atividades conjugadas, em grupos de pessoas, cuja frequência de aparecimento, nos depoimentos dos enfermeiros, foi de 70 vezes. As áreas temáticas de atuação encontradas no conteúdo analisado foram, na sua quase totalidade, a saúde do adulto em situações crônicas (hipertensão, diabetes) e saúde da mulher (na gravidez e no puerpério). Com relação à saúde da criança e à da pessoa idosa, ambas as áreas também foram enunciadas pelos entrevistados.

A educação em saúde na qual a prática clínica do enfermeiro ganha abrangência coletiva na organização do conhecimento na forma de atividade educativa, em seu significado mais abrangente da prática multidisciplinar, pode ser entendida como qualquer atividade, relacionada com aprendizagem, desenhada para alcançar saúde. ${ }^{9,11} \mathrm{Na}$ particularidade da Atenção Primária à Saúde, a educação tem se caracterizado como uma das principais estratégias para as mudanças de comportamento individuais e coletivas. Nesse conteúdo, o sentido da prática clínica, reiterou o já antes referido anteriormente, centrado, predominantemente, na necessidade de manutenção, mudança e aquisição de comportamentos mais saudáveis, a partir da aquisição de conhecimentos, atitudes e habilidades, acerca de processos de adoecimento.

O significado de prevenção ao risco do adoecimento, no sentido da mudança para um comportamento mais saudável, tem o componente cultural implícito às mudanças necessárias e está no próprio limite dos modos culturais das pessoas e das comunidades. Representa dizer que saber lidar com os agravos e processos de adoecimentos nas comunidades é assumir as diferenças culturais, não apenas em suas carências, mas na possibilidade de conhecer e visualizar suas potências para modos saudáveis de vida.

Nesse conteúdo da clínica, ele, ao organizar na forma de educação clínica, para aplicar no ob- jeto da ação, acrescenta um pressuposto filosófico e operativo de sentido, qual seja a troca de experiências e aderência aos aspectos culturais e sociais presentes na comunidade onde o indivíduo e a família vivem. Assim, é reforçado o pressuposto de a clínica com que o enfermeiro opera ser uma clínica própria, pois ele organiza este conhecimento, por exemplo, para a prevenção de doenças e agravos e para a promoção da saúde do indivíduo e da família.

Cabe salientar que também representa um marcador ideológico a contribuição da educação, seja reconhecida na atenção primária à saúde, na relação direta com as mudanças de estilos de vida ou estilos culturais com o propósito de uma vida mais saudável e constitui-se em estratégia difícil de ser avaliada, exatamente porque mudanças em estilos de vida são, em geral, alcançadas através da combinação de diferentes fatores da vida interagindo em conjunto. ${ }^{11}$

A prática clínica do enfermeiro tem, assim, tanto abrangência quantitativa, pela diminuição dos potenciais de riscos de adoecimento e sofrimento, quanto qualitativa, pela modificação dos estilos de vida dos indivíduos nas comunidades e, na intersecção quanti-qualitativa, efetua-se a relação com outro foco da raiz do conhecimento, com base populacional, no modelo de análise aplicado à comunidade e suas características coletivas, conforme é possível acompanhar na sequência do texto.

\section{DISCUSSÃO}

Por outro lado, reconhece-se a concomitância de matizes diferentes da organização do conhecer o objeto da clínica utilizada pelo enfermeiro na Atenção Primária à Saúde, na particularidade do estudo na saúde da família. O fato foi evidenciado com facilidade em algumas das narrativas em que a apreensão do objeto a se conhecer para a ação ficou na centralidade da clínica aplicada para intervir no limite do corpo orgânico e não pode ser entendido como um conhecimento orientador da prática do enfermeiro, somente, pois se estende para o campo da prática interdisciplinar, portanto, de outros profissionais. Isso no mínimo reduziria o que se tem de quantitativo na evidência qualitativa da prática do enfermeiro, um conhecimento da enfermagem que extrapola o limite de sua prática e é posto para o campo interdisciplinar, provocado pela própria natureza do objeto do conhecimento referido - a pessoa. A raiz disso encontra-se no conhecimento da prática clínica do enfermeiro e não de outro. Mas tal natureza provoca a ordem de 
organização do conhecimento da enfermagem, que é construída a partir daquela natureza do objeto de conhecimento de sua prática clínica: a ciência da enfermagem clínica.

A linha de demarcação entre o limite do conhecimento da prática clínica depende da objetividade do seu objeto de apreensão (sua objetividade exterior), realidade exterior a ser transformada pelo conhecimento e, ao mesmo tempo, a demarcação há pouco referida depende da experiência e da formação de cada uma das práticas que transforma seus objetos/sujeitos.

Por que, então, minimizar as características da ciência da Enfermagem que apreende seu objeto clínico de forma diferente de outro profissional, a exemplo dos achados deste estudo?

O que não é senão a realidade do objeto da ação - a pessoa na apreensão da prática clínica, organizado no limite possível do conteúdo da linguagem, posto na forma de conhecimento clínico da enfermagem que anuncia sua convicção científica, ou seja, a sua postura frente ao objeto do conhecimento de sua prática clínica.

Por conseguinte, permite-se contrariar alguns achados na literatura ${ }^{12-14}$ nos quais é reiterada a existência da centralidade da prática clínica de um único profissional, a saber, a prática clínica do médico, na organização do trabalho da SF. O que se evidenciou é uma centralidade no corpo e na linguagem que expressa o conhecimento posto na prática do conhecer o corpo clínico em questão. Salienta-se que o que se está reproduzindo - é uma das linguagens do conhecimento clínico e não se pode afirmar ser o único e verdadeiro conhecimento.

A linguagem reiterada pela coletividade científica pretende fortalecer o conhecimento da prática clínica como valor de troca, ou seja, como representante da valoração socialmente posta na prática de apenas um de seus representantes - a medicina clínica. Nele, a ideologia valorativa está embutida em si mesma, e essa dupla valorização possui raiz nas necessidades reconhecidas e apreendidas por meio da organização do conhecimento clínico e de sua aplicação.

O que difere, então, é a aplicação de um único conhecimento clínico? Isto significa que existe um único conteúdo clínico aplicado? Se a resposta for afirmativa, tem-se uma verdade que se reduz a uma única forma de organizar o conhecimento clínico do objeto e, portanto, redutora também do conhecimento em foco. Se a resposta for negativa, ou seja, existem diferentes formas de organizar o conhecimento clínico do objeto, tem-se uma negação da unificação. E, por consequência, a afirmação da incompletude das formas de conhecer um objeto que, por ser multifacetado tem, na tentativa de reduzi-lo a partes, findada sua constituição previsível, podendo aparecer outra(s) ordem(s) que não àquela(s) que deu(deram) origem à motivação do conhecer o objeto da clínica.

Objeto do conhecimento clínico e objeto da aplicação do conhecimento clínico: qual a diferença?

A valoração é assumida como verdade e, enquanto conhecimento verdadeiro, reafirma-se a valoração do mesmo na relação entre quem o utiliza para apreender o objeto de sua prática e ele próprio, o objeto da prática, que é o elemento "substância", o qual satisfaz a necessidade apresentada por ele próprio - o objeto da ação teórica (aplicação do conhecimento organizado em uma disciplina - sistemática) ou aplicação do conhecimento.

O objeto transformado por meio da descoberta feita pelo conhecimento aplicado, a prática clínica, reforça tal verdade socialmente valorada. Em outras palavras, o conhecimento clínico, aplicado à transformação/descoberta de seus objetos, reitera verdades anteriores e possibilita, pela natureza de sua constituição, refazer verdades de conhecer, ou de melhor conhecer, de estabelecer os limites entre o real verdadeiro e concreto e o real verdadeiro e abstrato.

Portanto, não se está questionando se é verdadeiro ou não o fato de o conhecimento da prática clínica do enfermeiro ser diferente; isso é algo que parte da própria matriz de constituição da ciência da enfermagem clínica, na qual o objeto a conhecer, a apreender é composto de elementos que conhecem a ele (objeto da ação) porque são colocados a se conhecer, são apreendidos porque descritos na matriz da apreensão, na matriz da aplicação teórica da prática clínica do enfermeiro. Também não se está negando ou simplesmente afirmando que o objeto da ação seja a pessoa em exata apreensão ou em pleno conhecimento. Entende-se que cada pessoa seja mais do que as fronteiras/limites apreendidos pela organização do conhecimento da clínica do enfermeiro.

Por outro lado, a tentativa apresentada aqui, de reiteração da ciência da Enfermagem pela apreensão do seu objeto clínico, não nega a intenção da valoração referida. E não nega, também, a ideologia premente e própria da característica da 
linguagem do conhecimento científico, pois ele é um modelo que se cria e se projeta sobre o real - a pessoa e sua clínica.

Outra pergunta necessita ser realizada, caso o que se tenha tentado demonstrar seja uma das formas de organizar o conhecimento clínico do enfermeiro na forma do objeto do conhecimento (objeto epistemológico) ou a sua aplicação (ainda objeto do conhecimento e seu desdobramento em objeto tecnológico) ao seu objeto da ação?

\section{RAZÃO INSTRUMENTAL - A MEDIDA DO POSSÍVEL DO CONHECIMENTO CLÍNICO}

Dito de outra forma, há certa dificuldade em prever o comportamento do todo - comunidade - e família, nos exemplos, a partir das propriedades de uma pessoa. Com efeito, por meio da utilização de modelos complexos, podese entender que a organização do conhecimento clínico em sua forma igualmente complexa auxilia a compreensão do enfermeiro a apreender o objeto da ação. É o modelo de organização que é importante para compreensão e explicação do todo, enquanto que as características das partes desempenham, com maior frequência, o papel coercivo, impondo limites aos desempenhos da organização, embora não produzam tais limites de uma forma direta.

Importante referir ainda que o conhecimento clínico complexo, mesmo auxiliando na compreensão e apreensão de uma coletividade, não necessariamente poderá ser prescritivo para um todo, por exemplo, a comunidade. ${ }^{1}$ Pode ser parte de uma descrição de base populacional, senão através de esquemas organizativos da razão, o conhecimento das características das partes, embora não seja suficiente para a compreensão das características desse todo. Mesmo sendo ele uma única pessoa ou um conjunto de pessoas, conforme o modo como foi referida a comunidade e seus grupos.

O que é feito, então? O enfermeiro utilizase do esquema da racionalidade instrumental, e organiza o conhecimento clínico de forma a poder ultrapassar o limite de uma pessoa. Ele organiza seu conhecimento (conhecer o objeto clínico) à maneira de um esquema operativo conjunto: os grupos temáticos que, motivados pelas necessidades sociais descritas nas políticas públicas, são acionados pelas mesmas necessidades demandadas de um perfil clínico-social reconhecido pelo conhecimento clínico complexo do enfermeiro.
Pode-se afirmar, assim, que o enfermeiro, no trabalho interdisciplinar da atenção primária, é o representante conflituoso do conhecimento clínico, pois na matriz de análise os três elementos da aplicação do conhecimento clínico constituídos, que são o indivíduo e a família, a comunidade e o sistema da atenção primária à saúde, na particularidade na saúde da família, foram estudados na relação entre a racionalidade instrumental e o objeto da prática, no qual é aplicada a referida racionalidade.

\section{REFERÊNCIAS}

1. Swearingen CD. Using nursing perspectives to inform public health nursing workforce development. Public Health Nurs. 2009 Jan-Feb; 26(1):79-87.

2. Raghupathi W. Designing clinical decision support systems in health care: a systemic view. Int J Healthc Inf Syst Inform. 2006 May-Aug; 2(1):44-53.

3. Lauri S, Salanterä S, Bild H, Chalmers K, Duffy M, Kim HS, et al. Public health nurses' decision making in Canada, Finland, Norway, and the United States. West J Nurs Res. 1997April; 19(2):143-65.

4. 4 Carr SM. Framework for understanding clinical reasoning in community nursing. J Clin Nurs. 2004 Oct; 13(7):850-57.

5. Lefebvre H. Lógica formal, lógica dialética. $6^{a}$ ed. São Paulo (SP): Civilização Brasileira, 1995.

6. Green J, Thorogood N. Qualitative methods for health research. London (UK): Sage, 2005.

7. Edwards SD. What is philosophy of nursing? J Adv Nurs. 1997 May; 25(5):1089-93.

8. Cezar-Vaz MR, Cabreira GO, Couto ZFS, Soares JFS, Weis AH, Berto J. O trabalho da enfermeira na Atenção Básica de Saúde: assumindo uma forma programática para o conteúdo clínico-social. Texto Contexto Enferm. 2003 Jul-Set; 12(3):342-50.

9. Baldwin JH, Conger CO, Abegglen JC, Hill EM. Population-focused and community-based nursingmoving toward clarification of concepts. Public Health Nurs. 1998 Feb; 15(1):12-8.

10. Ministério da Saúde (BR). Portaria No 648, de 28 de Março de 2006: aprova a Política Nacional de Atenção Básica, estabelecendo a revisão de diretrizes e normas para a organização da Atenção Básica para o Programa Saúde da Família e o Programa Agentes Comunitários de Saúde. Diário Oficial da União. República Federativa do Brasil, 29 Mar 2006.

11. Bramadar IJ, Chalmers K, Andrusyszyn MA. Knowledge, skills and experiences for community health nursing practice: the perceptions of community nurses, administrators and educators. J Adv Nurs. 1996 Dec; 24:1224-33.

12. Barboza TAV, Fracolli LA. A utilização do "fluxograma analisador" para a organização da 
assistência à saúde no Programa Saúde da Família. Cad Saúde Pública. 2005 Jul-Ago; 21(4):1036-44.

13. Rocha PM, Uchoa AC, Rocha NSPD, Souza ECF, Rocha ML, Pinheiro TXA. Avaliação do Programa Saúde da Família em municípios do Nordeste brasileiro: velhos e novos desafios. Cad Saúde Pública. 2008; 24(Sup.1):S69-78.

14. Alves VS. Educação em saúde e constituição de sujeitos: desafios ao cuidado no Programa Saúde da Família [dissertação]. Salvador (BA): Universidade da Bahia. Instituto de Saúde Coletiva; 2004. 